\title{
Low-dimensional offshore wave input for extreme event quantification
}

\author{
Šehi, Kenan; Bredmose, Henrik; Sørensen, John D.; Karamehmedovi, Mirza
}

Published in:

Journal of Engineering Mathematics

Link to article, DOI:

10.1007/s10665-021-10091-w

Publication date:

2021

Document Version

Peer reviewed version

Link back to DTU Orbit

Citation (APA):

Šehi, K., Bredmose, H., Sørensen, J. D., \& Karamehmedovi, M. (2021). Low-dimensional offshore wave input for extreme event quantification. Journal of Engineering Mathematics, 126(13). https://doi.org/10.1007/s10665-021$10091-w$

\section{General rights}

Copyright and moral rights for the publications made accessible in the public portal are retained by the authors and/or other copyright owners and it is a condition of accessing publications that users recognise and abide by the legal requirements associated with these rights.

- Users may download and print one copy of any publication from the public portal for the purpose of private study or research.

- You may not further distribute the material or use it for any profit-making activity or commercial gain

- You may freely distribute the URL identifying the publication in the public portal

If you believe that this document breaches copyright please contact us providing details, and we will remove access to the work immediately and investigate your claim 


\title{
Low-dimensional offshore wave input for extreme event quantification
}

\author{
Kenan Šehić $\mathbb{D} \cdot$ Henrik Bredmose • \\ John D. Sørensen • Mirza Karamehmedović
}

Received: 6 January 2020 / Accepted: 5 January 2021

(C) The Author(s), under exclusive licence to Springer Nature B.V. part of Springer Nature 2021

\begin{abstract}
In offshore engineering design, nonlinear wave models are often used to propagate stochastic waves from an input boundary to the location of an offshore structure. Each wave realization is typically characterized by a high-dimensional input time-series, and a reliable determination of the extreme events is associated with substantial computational effort. As the sea depth decreases, extreme events become more difficult to evaluate. We here construct a low-dimensional characterization of the candidate input time series to circumvent the search for extreme wave events in a high-dimensional input probability space. Each wave input is represented by a unique low-dimensional set of parameters for which standard surrogate approximations, such as Gaussian processes, can estimate the short-term exceedance probability efficiently and accurately. We demonstrate the advantages of the new approach with a simple shallow-water wave model based on the Korteweg-de Vries equation for which we can provide an accurate reference solution based on the simple Monte Carlo method. We furthermore apply the method to a fully nonlinear wave model for wave propagation over a sloping seabed. The results demonstrate that the Gaussian process can learn accurately the tail of the heavy-tailed distribution of the maximum wave crest elevation based on only $1.7 \%$ of the required Monte Carlo evaluations.
\end{abstract}

Keywords Dimensionality reduction · Extreme events · Gaussian process · Offshore applications · Sequential design

\section{Introduction}

The occurrence of extreme events is typically quantified by a $d$-fold integral, called the probability of failure,

$P_{\mathrm{F}}=\int_{\gamma-g(\theta) \leq 0} \pi_{d}(\theta) \mathrm{d} \theta$,

K. Šehić $(\varangle) \cdot$ M. Karamehmedović

Department of Applied Mathematics and Computer Science, Technical University of Denmark, 2800 Kgs Lyngby, Denmark e-mail: kense@dtu.dk

H. Bredmose · J. D. Sørensen

Department of Wind Energy, Technical University of Denmark, 2800 Kgs Lyngby, Denmark

J. D. Sørensen

Department of Civil Engineering, Aalborg University, 9100 Aalborg, Denmark 
where $\theta \in \mathbb{R}^{d}$ is the stochastic input of a limit-state function $\gamma-g(\theta), \pi_{d}$ is the joint probability density function (PDF) for $\theta$ and $g(\theta) \geq \gamma$ defines a failure event with a failure threshold $\gamma$ for an arbitrary function (i.e., a numerical model) $g: \mathbb{R}^{d} \rightarrow \mathbb{R}$. In the present study, the failure event is defined by a wave crest elevation $g(\theta)$ (for example, the maximum 1-h crest elevation) exceeding a certain threshold $\gamma$. We recognize the probability of failure, Eq. (1.1), as the short-term exceedance probability since the failure event is defined for a predefined sea state. For a real design situation, the short-term probability distribution would be further integrated over the range of possible sea states to define the long-term exceedance probability. We here focus only on the efficient evaluation of the short-term exceedance probability. We define $\theta$ to be a highly dimensional vector of stochastic Fourier coefficients that generate input waves which recreate real offshore conditions [1-4].

In offshore engineering, the short-term exceedance probability, Eq. (1.1), is often estimated with the well-known reliability approaches FORM/SORM, which are the first/second-order Taylor series approximations of the limitstate function at the design point. However, FORM/SORM idealize the failure surface and fall short of defining multiple failure regions [5]. Additionally, as a geometrical approach, FORM/SORM do not provide error estimates. A robust alternative is the simple Monte Carlo (MC) method [6]. It does not depend on the dimension of the input and can find multiple design points for almost any numerical model. In this framework, the short-term exceedance probability, Eq. (1.1), is defined as the sample mean of the indicator function $\mathbb{I}(\theta)$, where $\mathbb{I}(\theta)=1$ if $g(\theta) \geq \gamma$ and $\mathbb{I}(\theta)=0$ otherwise. The idea is to compute $N$ blind samples of the numerical model and estimate the sample mean. While being a straightforward approach to implement, $\mathrm{MC}$ is infeasible in conjunction with expensive numerical models due to the slow convergence rate $\mathcal{O}\left(N^{-1 / 2}\right)[6]$. As we are only able to approximate the sample mean Eq. (1.1), we employ the mean squared error measure to define a sufficient number of evaluations $N$ for a prescribed relative error [6]. For example, the response value of an exceedance probability of $2 \times 10^{-3}$ with the relative error less than 0.1 requires at least $N=5 \times 10^{4}$ evaluations. A numerical model which needs 1 min to compute would require approximately 35 days to find the short-term exceedance probability. Therefore, surrogate methods such as Polynomial Chaos expansion [7] and Gaussian (Kriging) process [8] are usually proposed to define a low-cost surrogate for the numerical model using a small number of evaluations. The low-cost surrogate is then employed to approximate efficiently the short-term exceedance probability. However, the requirements for standard surrogate methods typically exponentially increase with the dimension due to the curse of dimensionality [9]. For high-dimensional problems, as the one considered in this paper, this approach becomes infeasible.

Alternative approaches consider only the statistical characteristics of extreme events such as extreme value theorems [10] and large deviations theory [11,12]. However, these methods have essential limitations requiring various extrapolation schemes due to the insufficient size of the sample set, and they therefore cannot always explain the nontrivial shape of the tail. The Fokker-Planck equation (FPE) [13] incorporates into the estimation the governing dynamical system of the model, as well as the stochastic nature of extreme events. However, even in low-dimensional cases, the FPE is prohibitively difficult to solve.

Mohamad et al. [14] proposed a sequential sampling strategy based on Gaussian process regression and statistical properties of extreme events. They formulated an optimization process that selected the most informative design point according to the log-L2 criterion, which eventually reduce the uncertainties in the prediction of extreme events. To avoid the curse of dimensionality, their numerical implementation is low-dimensional. For a wave-propagation application, they characterize the input condition by just two parameters, namely wave group length and amplitude. Although the wave group parameters are good indicators of extreme wave events [15-20], the propagation over long distances from the generation boundary makes single-group characteristics less relevant in the general case. Other options would be to employ standard dimensionality reduction tools such as principal component analysis (PCA) [21,22], partial least-squares regression (PLS) [23,24], active-subspace analysis [9,20], or autoencoders [25]. However, these standard dimensionality reduction tools may provide inefficient reduction in offshore applications or suffer from expensive and intrusive implementation.

To improve on this, we propose a low-dimensional classification representation of the high-dimensional Gaussian input. Based on the input sample set of the predefined input sea state, we classify the wave input using classification parameters unique for each time series. This technique is typically recognized as feature extraction [26]. The objective is to describe correlated data, such as a time-series or an image, by a few standard statistical measures 
such as variance or entropy. These classification parameters are now the low-dimensional design input parameter for data-driven model such as Gaussian process regression. To reduce the prediction uncertainties for extreme events, we select the most informative design points from the input Gaussian evaluations based on the probability of misclassification [8]. We test our approach in two numerical experiments. The first is based on the Korteweg-de Vries equation, which is a simple shallow-water wave model for which we can generate the reference solution with the simple Monte Carlo method. The second case is fully nonlinear wave propagation over a sloping seabed, computed with the OceanWave3D model [27]. We select the classification parameters heuristically, and view this paper as initial work on the low-dimensional representation of offshore wave input, or generally of input time-series for any system.

Section 2 covers Gaussian process regression in general. We introduce the low-dimensional representation of input time-series in Sect. 3, and present the numerical experiments in Sect. 4. There, we demonstrate that the lowdimensional representation based on our classification parameters is suitable for the Gaussian process design and efficient quantification of extreme events. We present our conclusions in Sect. 5.

\section{Gaussian process regression}

When the numerical cost of a model prohibits the quantification of the involved uncertainties, it is natural to try to construct a surrogate model, a low-cost replacement based on a small number of evaluations $N_{\mathrm{GP}}$ of the original model. In this work we use Gaussian or Kriging process regression (GP), which employs the Gaussian distribution over a training set based on a Bayesian approximation [28]. The training set, $\mathcal{S}=\left\{\left(\theta_{i}, g\left(\theta_{i}\right)\right)\right\}$, includes the matrix of the input parameter $\mathbf{X}=\left(\theta_{i}\right) \in \mathbb{R}^{N_{\mathrm{GP}} \times d}$ and the corresponding evaluations of a numerical model $Y=\left(Y_{i}=g\left(\theta_{i}\right)\right) \in \mathbb{R}^{N_{\mathrm{GP}} \times 1}$. For an arbitrary smooth function $g$, we define a Gaussian process approximation by $[8,28]$

$g(\theta) \approx \hat{g}(\theta)=\beta^{\mathrm{T}} \cdot f_{\mathrm{T}}(\theta)+\sigma^{2} Z\left(\theta, \omega_{z}\right)$,

where $\beta^{\mathrm{T}} \cdot f_{\mathrm{T}}(\theta)$ is the trend, $\sigma^{2}$ is the variance of the Gaussian process, and $Z\left(\theta, \omega_{z}\right)$ is a zero-mean, unit-variance stationary Gaussian process with $\omega_{z} \in \Omega$ as a sample point in the probability space $(\Omega, \mathcal{F}, \mathbb{P})$. The trend $f_{\mathrm{T}}(\theta)$ describes the global behavior of the training set, and is defined using simple regression. The complexity of the Gaussian process $Z$ is described by a stationary kernel matrix $\mathbf{K}_{i j}=K\left(\left|\theta_{i}-\theta_{j}\right| ; \Theta\right)$, where $\Theta$ are the hyperparameters such as the overall correlation of the samples or the smoothness of the training set. The hyperparameters $\Theta$ for the kernel matrix $\mathbf{K}_{i j}$ are found using maximum likelihood estimation, i.e., maximizing the log marginal likelihood of the training set [29]

$\log p(Y \mid X, \Theta)=-\frac{1}{2}\left(Y-\mathbf{F}_{\mathrm{T}} \beta\right)^{\mathrm{T}} \mathbf{C}^{-1}\left(Y-\mathbf{F}_{\mathrm{T}} \beta\right)-\frac{1}{2} \log (\operatorname{det} \mathbf{C})-\frac{N}{2} \log (2 \pi)$.

Here we define the matrix $\mathbf{C}$ that depends on the hyperparameters $\Theta$ as $\mathbf{K}+\sigma \mathbf{I}$, while $\mathbf{F}_{\mathrm{T}}$ is the information matrix, i.e., the regression estimation with respect to the global behavior of the training set. Thus, an arbitrary smooth function $g$ is eventually marginalized out. The first term of Eq. (2.2) is a Mahalanobis distance between the predictions of the model and the training set, i.e., the quality of the model to reproduce the data. The complexity of the model is described by the second term. A smoother covariance matrix $\mathbf{C}$ has a smaller determinant, which generates a lower penalty [29]. With larger training sets $N$, the likelihood, Eq. (2.2), is reduced due to the last term, which is a function of $N$. While a simple regression assumes the errors of predictions to be independent, Gaussian process regression via a stationary kernel matrix and adequate hyperparameters integrates the correlation within the training set to describe the interaction between different observations. Hence, the performance of the GP regression depends highly on the selection of the kernel function. For example, a periodic kernel may be best suited for a 
periodic training set. We define the regression parameters $\beta$ and $\sigma^{2}$ based on generalized least-squares regression [8]. A typical challenge is to select an optimal size $N_{\mathrm{GP}}$ of the training set. Gramacy and Apley [30] propose to select the training size by adding observations until a certain mean-squared predictive error is no longer fulfilled. Once the GP model is trained, we estimate for an arbitrary input sample $\theta^{*}$ the prediction $\mu_{g}\left(\theta^{*}\right)$ and the variance (i.e., an uncertainty measure) $\sigma_{g}^{2}\left(\theta^{*}\right)$ via $[8,28]$

$\mu_{g}\left(\theta^{*}\right)=f_{\mathrm{T}}\left(\theta^{*}\right) \cdot \beta+k\left(\theta^{*}\right)^{\mathrm{T}} \mathbf{K}^{-1}\left(Y-\mathbf{F}_{\mathrm{T}} \beta\right)$,

and

$\sigma_{g}^{2}\left(\theta^{*}\right)=\sigma^{2}\left(1-\left\langle f_{\mathrm{T}}\left(\theta^{*}\right)^{\mathrm{T}} k\left(\theta^{*}\right)^{\mathrm{T}}\right\rangle\left[\begin{array}{cc}0 & \mathbf{F}_{\mathrm{T}}^{\mathrm{T}} \\ \mathbf{F}_{\mathrm{T}} & \mathbf{K}\end{array}\right]^{-1}\left[\begin{array}{c}f_{\mathrm{T}}\left(\theta^{*}\right) \\ k\left(\theta^{*}\right)\end{array}\right]\right)$.

Here $k\left(\theta^{*}\right)$ is the correlation between the arbitrary input sample and the rest of the samples within the training set.

\subsection{Sequential uncertainty reduction for extreme events}

Gaussian process regression provides the posterior distribution, based on the moments (2.3) and (2.4), which describes the quality of the predictions and, in general, the performance of the surrogate model. An arbitrary Gaussian prediction $\mu_{g}\left(\theta^{*}\right)$ and the corresponding standard deviation $\sigma_{g}\left(\theta^{*}\right)$ define the confidence interval $(\mathrm{CI})$ $[8,28]$

$\mu_{\mathrm{CI}}^{ \pm}\left(\theta^{*}\right)=\mu_{g}\left(\theta^{*}\right) \pm \alpha \cdot \sigma_{g}\left(\theta^{*}\right)$,

with some confidence level $\alpha$. For example, $\alpha=1.96$ for $95 \%$ confidence, and $95 \%$ of the area of the normal distribution is within 1.96 standard deviations. Narrower confidence intervals mean more confidence in the predictions and generally in the performance of the surrogate model, under the assumption that the limit-state function is smooth. However, a significantly narrow CI can still over/under-predict a highly nontrivial response, because Gaussian process regression is a design method. The training set and the initial design may not be sufficient to accurately describe a complicated function. The application is not straightforward and should be employed carefully with an understanding of the numerical model and the data itself. Hence, the idea is to sequentially design numerical experiments $\theta^{*}$ for training while exploring optimally the probability space (i.e., using a learning function) to create an optimal Gaussian process. Mohamad et al. [14] proposed a sequential design utilizing the log-L2 distance between the upper and lower bound of CI to reduce uncertainties for extreme events. Schöbi et al. [8] used the probability of misclassification to define which numerical experiments $\theta^{*}$ are close to a predefined failure threshold or are poorly predicted. We find this sufficient for our analysis.

As the probability of failure essentially involves a binary classification, with 1 for failure and 0 otherwise, the misclassification of predictions based on the first two moments and on the failure threshold $\gamma$ is a natural option for the Gaussian process regression. We write for the probability of misclassification $P_{\mathrm{M}}$ [8]

$P_{\mathrm{M}}\left(\theta^{*}\right)=\Phi\left(-\frac{\left|\mu_{g}\left(\theta^{*}\right)-\gamma\right|}{\sigma_{g}\left(\theta^{*}\right)}\right)$

Here, the $U$-function (the learning function) is the fraction in the argument in (2.6). It is recognized as the reliability index attached to $P_{\mathrm{M}}$ with $\Phi$ as the cumulative distribution function of the standard normal distribution. Small values of the $U$-function reveal the samples $\theta^{*}$ that are close to the predefined failure threshold $\gamma$ or have high 
uncertainties in predictions. Therefore, to improve the training set, we select the most informative design points, i.e., the input parameter $\theta^{*}$ drawn from the joint $\operatorname{PDF} \pi_{d}$ with the smallest $U$-values for the trained Gaussian process [8]

$\theta^{*}=\arg \min _{\theta \in \mathbb{R}^{d}} \frac{\left|\mu_{g}(\theta)-\gamma\right|}{\sigma_{g}(\theta)}$.

The procedure is iterative, as we add more $U$-based design points $\theta^{*}$ until an error measure based on the confidence interval for the failure level drops below some prescribed threshold as demonstrated in [8]. As the trained GP is a surrogate model, the optimization procedure is low cost. This produces a Gaussian process designed specially for extreme events. However, in the present study, the optimization procedure within (2.7) is based on the generated

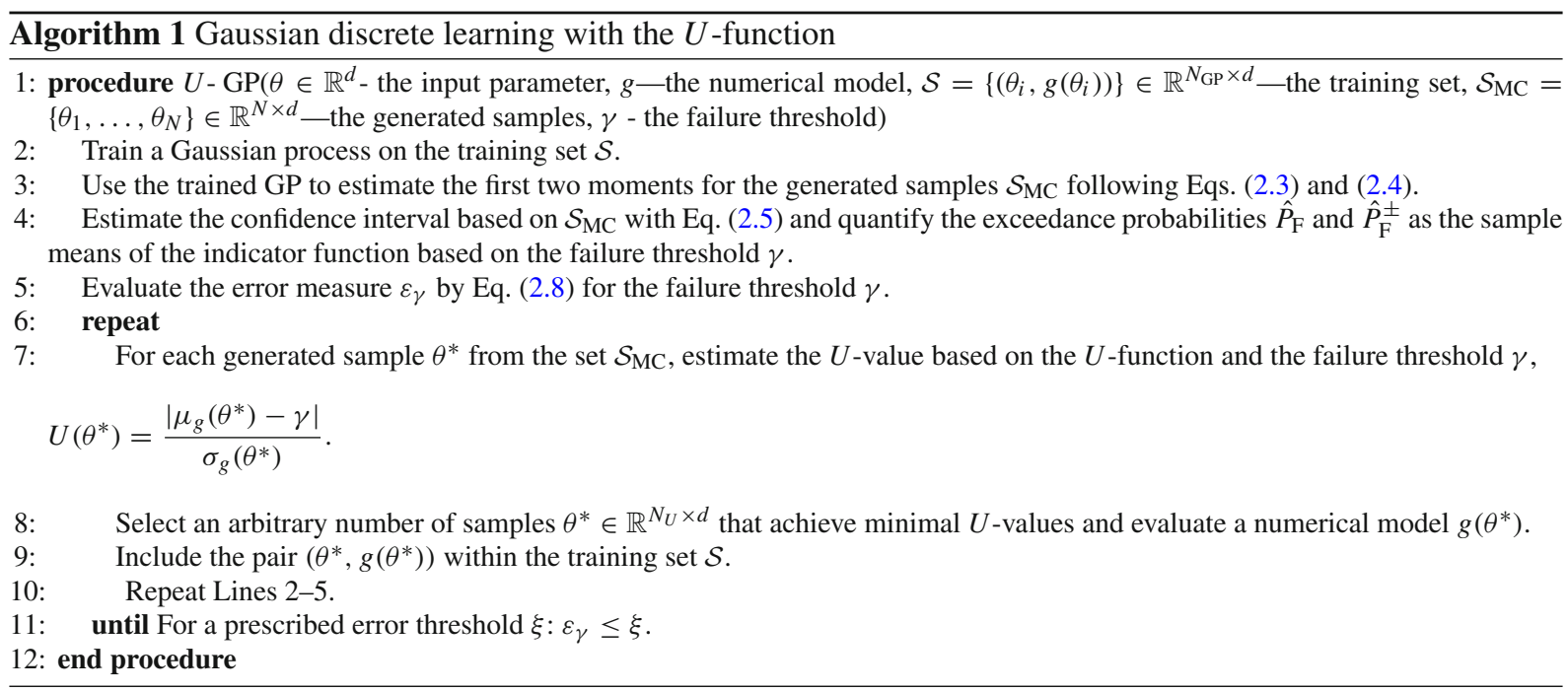

samples $\mathcal{S}_{\mathrm{MC}}=\left\{\theta_{1}, \ldots, \theta_{N}\right\} \in \mathbb{R}^{N \times d}$ that we utilize to define a low-dimensional representation of the original input parameter $\theta$ as explained in the next section. It can be seen as discrete learning, since we rely only on the generated samples $\mathcal{S}_{\mathrm{MC}}$. The procedure is described in more detail in Algorithm 1. The learning is terminated once the error measure, a stopping criterion, is fulfilled, which we define as [8]

$\varepsilon_{\gamma}=\frac{\left|\hat{P}_{\mathrm{F}}^{+}-\hat{P}_{\mathrm{F}}^{-}\right|}{\hat{P}_{\mathrm{F}}}$,

where the exceedance probabilities $\hat{P}_{\mathrm{F}}$, Eq. (1.1), and $\hat{P}_{\mathrm{F}}^{ \pm}$are estimated as the sample means of the indicator function by the simple Monte Carlo method based on the generated samples $\mathcal{S}_{\mathrm{MC}}$. The exceedance probabilities $\hat{P}_{\mathrm{F}}^{ \pm}$involve the upper and lower bounds of the confidence interval. The error measure (2.8) usually ranges between 0.5 and 2 [8]. It depends on the final failure level and the accuracy requirement. If the error measure is not achieved (i.e., $\varepsilon_{\gamma}>\xi$ ), the discrete learning converges to the simple Monte Carlo estimation of the generated samples. To additionally improve the estimation of the probability of failure, one can iteratively replace approximations close the failure surface with corresponding numerical evaluations until the difference between two consecutive estimations of the probability of failure is fulfilled [7]. 


\section{Low-dimensional representation}

When the input dimension $d$ is large, e.g., $d=100$, it is required to define a larger training set $N_{\mathrm{GP}}$ based on the factorial design. However, the computation of the Gaussian process becomes impractical as an $N_{\mathrm{GP}} \times N_{\mathrm{GP}}$ kernel matrix needs to be inverted several times to make an arbitrary prediction, which costs $\mathcal{O}\left(N_{\mathrm{GP}}^{3}\right)$. Therefore, the literature on Gaussian process regression typically covers low-dimensional numerical implementations [14,17].

In our work, related to offshore applications, the input parameter is defined within a high-dimensional probability space to recreate the environmental conditions accurately. The boundary condition, which we note as the wave input at the spatial location $x=0$, is defined as a Fourier series with multi-dimensional random coefficients drawn from the standard normal distribution (i.e., $\mathcal{N}(0,1))[31]$

$$
\eta_{\mathrm{BC}}:=\eta(x=0, t)=\sum_{j=1}^{d / 2} \sqrt{S\left(f_{j}\right) \cdot \Delta f}\left[A_{j} \cos \left(2 \pi f_{j} t-k_{j} x\right)+B_{j} \sin \left(2 \pi f_{j} t-k_{j} x\right)\right] .
$$

Here $S\left(f_{j}\right)$ is the wave energy spectrum, i.e., JONSWAP [4], $f_{j}$ are wave frequencies, $k_{j}$ are the wavenumbers and $A_{j}$ and $B_{j}$ are random variables drawn from $\mathcal{N}(0,1)$. To simplify the notation, the input parameter $A_{j}$ and $B_{j}$ are written as $\theta=\left(A_{1}, \ldots, A_{d / 2}, B_{1}, \ldots, B_{d / 2}\right) \in \mathbb{R}^{d}$. The dimensionality $d$ is derived from the discretization of the frequency range with the frequency step $\Delta f=1 / T$, where $T$ is the time duration of the numerical simulation. Each spectrum area $S\left(f_{j}\right) \Delta f$ that corresponds to a certain frequency value $f_{j}$ is multiplied with random variables $A_{j}$ and $B_{j}$. For example, for 1-h wave propagation and the frequency range from $0.05 \mathrm{~Hz}$ up to $0.3 \mathrm{~Hz}$, we have $d=1802$. This is an extreme problem of uncertainty quantification due to the curse of dimensionality, which typically makes the problem complexity grow exponentially with the dimension. Also, in offshore applications, the numerical model usually imposes intensive computation and is to be employed only a limited number of times [14].

We propose a novel approach to quantify extreme events for reliability analysis in otherwise infeasible situations. The idea is to utilize the correlation of the boundary condition, Eq. (3.1), which can be recognized as a modified white noise (i.e., a time-series), to define a low-dimensional representation for data-driven models such as Gaussian process regression. Feature extraction [26] is a standard classification approach in machine learning, which is used to transform patterns into features to reduce the dimensionality of correlated data. In [26], feature extraction is applied to define a low-dimensional representation of drilling time-series data. In general, each correlated timeseries has a unique set of characteristics. Therefore, the selected features such as variance, entropy, and similar are then employed to train a classifier (e.g., a support vector machine) on the low-dimensional representation for the final pattern recognition. Due to a smaller feature space, the computation load for a classifier is significantly reduced with adequate predictions.

In the present study, by using feature extraction [26], our original problem set can be changed from the extreme high-dimensional Fourier coefficients $\theta$ to the low-dimensional representation based on certain classification parameters $K$ of the input surface elevation equation (3.1). Many statistical features can be employed to measure different properties of the initial surface elevation such as to measure variability, shape or central tendency. Here, we choose the standard statistical measures found also in [26]. First, we generate $N$ number of input surface elevations, Eq. (3.1), for the time duration $T$ to define the time-series data used for feature extraction. The number of input surface elevations (i.e., time-series) $N$ corresponds to the simple Monte Carlo requirement based on the mean-squared estimation and the targeted exceedance probability. As we have mentioned previously, the exceedance probability of $2 \times 10^{-3}$ would require $N=5 \times 10^{4}$ initial surface elevations to fulfill a relative error of $10 \%$. Therefore, with the generated time-series data, we define the low-dimensional representation of the wave input heuristically based on 10 standard features (i.e., classification parameters) $K_{1-10}$ (scaled by their maximum values) as [26]

- $K_{1}:=\max \left(\eta_{\mathrm{BC}}\right)$ - the maximum crest elevation of the wave input.

- $K_{2}$-the wave input variance-second moment $\sigma^{2}$.

- $K_{3}$ - the wave input skewness - third moment. 
- $K_{4}$ - the wave input kurtosis - fourth moment.

- $K_{5}$ - the wave input root mean square (RMS).

- $K_{6}$ - the wave input approximate entropy, which measures complexity.

- $K_{7}$ - the wave input percentile for $50 \%$.

- $K_{8}$ - the wave input percentile for $75 \%$.

- $K_{9}$ - the wave input percentile for $90 \%$.

- $K_{10}$ - the wave input mode as the most frequent value within the surface elevation.

Evidently, each additional feature would improve an approximation of a time-series (i.e., the boundary condition Eq. (3.1)) and eventually learning. However, as a part of stochastic modeling, the objective is to reduce the computation load by providing a low-dimensional space for learning. We select these 10 standard features as the adequate balance between sufficient feature extraction and the computational cost of stochastic modeling such as Gaussian process regression. We do not claim that these 10 features are optimal. For example, $K_{2}$ and $K_{5}$ are directly related for a zero-mean signal. Our focus here is on examining the application of standard feature extraction to quantify extreme events in stochastic modeling and provide a basis for future developments. In addition, an expert's experience and real-time experiments could be potentially used to improve a low-dimensional representation by identifying essential characteristics of the wave input with respect to the quantity of interest.

\section{Numerical experiments}

We illustrate the proposed approach on two offshore problems. The first example uses the modified Korteweg-de Vries equation (KdV22) [20]. The second application, involving a simple OceanWave3D benchmark with wave propagation over a slope [32], demonstrates the applicability of the proposed classification approach to a fully nonlinear model. As the computations are intensive, the quantification of extreme events using standard methods is infeasible. Generally, we assume that the computational budget is limited.

The quantity of interest is the maximum crest elevation $\eta_{\max }$ at the reference location $x^{*}$ for an offshore structure,

$\eta_{\max }=\max \left\{\eta\left(x^{*}, t\right), 0 \leq t \leq T\right\}$.

The objective is to estimate the short-term exceedance probability for $\eta_{\max }$ based on a predefined sea state. For both cases, we employ the JONSWAP spectrum, which is typically used for extreme events analyses. Due to the computation limitations, we employ short numerical simulations to examine the advantages and the disadvantages of our approach. The calculations are executed on a personal laptop with Intel Core i5-6200U CPU @ 2.30GHz $\times$ 4.

\subsection{A simple shallow-water wave model}

Let us consider unsteady water waves defined by the Korteweg-de Vries equation (KdV) for one-dimensional nonlinear surface flows under the influence of gravity [20,33],

$$
\eta_{t}(x, t)+\sqrt{g \cdot h} \cdot \eta_{x}(x, t)+\frac{3}{2} \sqrt{\frac{g}{h}} \eta(x, t) \eta_{x}(x, t)+\left(\beta+\frac{1}{6}\right) \sqrt{\frac{g}{h}} h^{3} \eta_{x x x}(x, t)+\beta h^{2} \eta_{x x t}(x, t)=0 .
$$

Here $\eta(x, t)$ is the free surface elevation, measured upwards from the still water level, $\beta=19 / 60, h$ is the seabed depth, $x$ is the horizontal coordinate, $t$ is time, and $g$ is the gravitational acceleration. The flow is assumed to be inviscid and irrotational, and the seabed flat with a depth of $h=20 \mathrm{~m}$. We propagate waves for $T=600 \mathrm{~s}$ with $\Delta t=0.0824 \mathrm{~s}$ and a high-cut frequency value of $0.3 \mathrm{~Hz}$. The present version of the equation with $\beta=19 / 60$ provides improved dispersion properties with a Padé[2,2] fit of the linear phase speed. We solve Eq. (4.2) by 
(a) 15

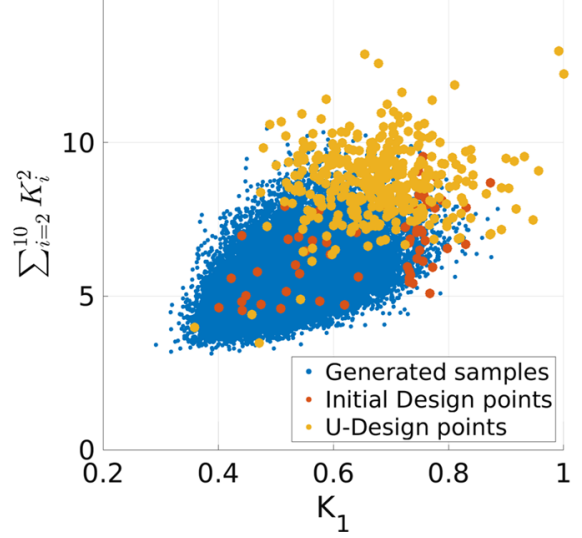

(b) 15

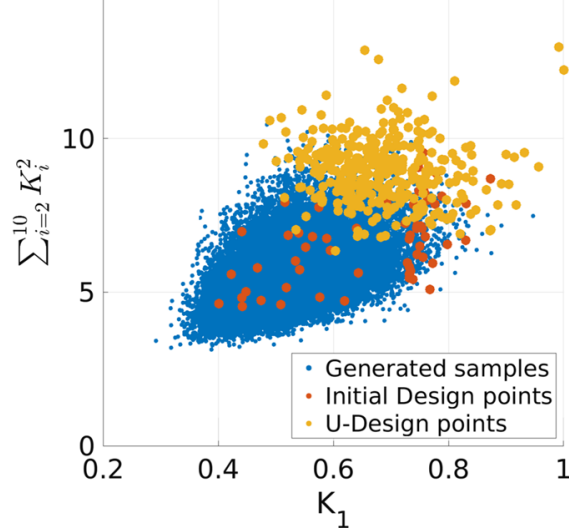

Fig. 1 The wave input classification data generated using $H_{\mathrm{S}}=6.8 \mathrm{~m}$ and $T_{\mathrm{P}}=15 \mathrm{~s}$. a $U$-design points for the pure quadratic trend, and $\mathbf{b} U$-design points for the linear trend. (Color figure online)

splitting temporal and spatial derivatives. Along the spatial axis, the problem is extended periodically [20]. The spectral content above $60 \%$ of the Nyquist wave number is disregarded to cancel aliasing. Hence, the classical fourth-order Runge-Kutta approach is employed for the time integration. We select $H_{\mathrm{S}}=6.8 \mathrm{~m}$ and $T_{\mathrm{P}}=15 \mathrm{~s}$, as representative values of a 100 -year return period.

First, based on the simple Monte Carlo requirement for the target exceedance probability, we generate $N=5 \cdot 10^{4}$ wave input samples with the predefined sea state. For each wave input, we calculate the scaled classification parameters $K_{1-10}$ as previously explained. To visualize the time-series data in a two-dimensional figure, we plot along the vertical axis of Fig. 1 the sum of the squares of the classification parameters, and the scaled classification parameter $K_{1}$ of the input maximum crest elevation $\eta_{\max }(0, t)$ along the horizontal axis. Each blue sample is one wave input (i.e., time-series) for a matching vector of $\theta$ drawn from $\mathcal{N}(0,1)$. In Fig. 1, orange points (i.e., initial design points) are used for initial training of a surrogate model, while yellow points (i.e., $U$-design points) are used for active learning. Figure 1 shows the correlation between $K_{1}$ and the squared sum of the classification parameters. It is similar to the correlation between the wave group amplitudes and lengths in the work of Mohamad et al. [14].

Figure 2a shows the Pearson correlations between the classification parameters. It is worth noting that the variance factor $K_{2}$ has a significant positive correlation with the root mean-squared factor $K_{5}$ and the percentile parameters for $75 \%$ and $90 \%\left(K_{8}\right.$ and $\left.K_{9}\right)$ as expected, while it has a significant negative correlation with the approximated entropy factor $K_{6}$. The term correlation here concerns the intensity and direction of the linear relationship between two parameters. For perfectly correlated features, only one feature should be used for the analysis as the overall performance would not be affected. In addition, stochastic modeling would require less numerical evaluations for a smaller dimension of the problem. However, even insignificant difference can provide an important leverage for discrete learning to efficiently quantify extreme events. Rather than disregarding nearly correlated features, a suitable option would be to use PCA to examine the total variation of eigenvalues and adequately define a low-dimensional subspace, which is beyond the scope of this work. We find the global sensitivity of the 10 classification parameters $K_{1-10}$ using the Pearson correlation and $N$ reference evaluations of the KdV22 model as provided in Fig. $2 \mathrm{~b}$. The most important parameter turns out to be the most frequent value $\left|K_{10}\right|$ within the wave input time-series. Typically, in offshore engineering, the skewness is recognized to be important for the quantification of extreme events. In this case, the relation is insignificant. We find that the least relevant parameter is the time-series percentile for $50 \%$, with virtually zero Pearson correlation.

Using the factorial design, the construction of a quadratic response surface requires at least $N_{\mathrm{GP}}=(d+1)(d+2) / 2$ observations [34]. We use this relation as the reference point for the Gaussian process design. For the 10-dimensional case, the number of samples is $N_{\mathrm{GP}}=66$. However, as we focus on the sequential improvement of Gaussian models, 
(a)

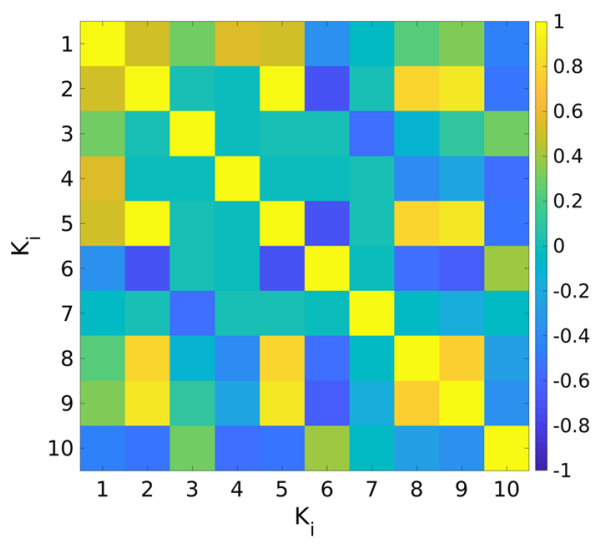

(b)

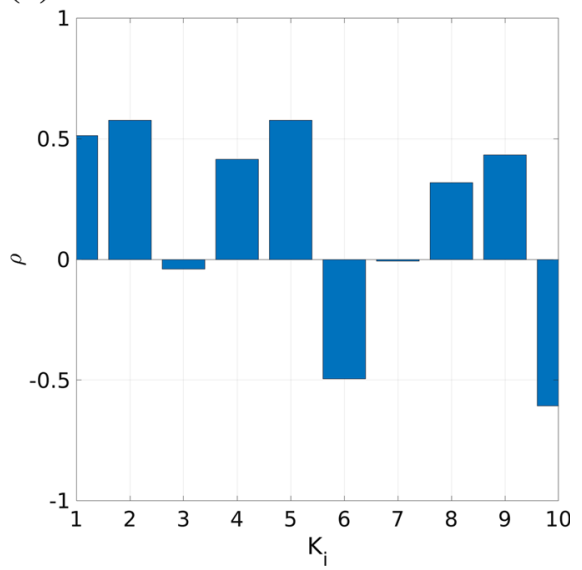

Fig. 2 a The correlation matrix for the wave generation classification parameters $K_{1-10}$ at $x=0$. b The correlation relations between the quantity of interest $\eta_{\max }$ and the classification parameters $K_{1-10}$ at the reference location $x=x^{*}$. (Color figure online)
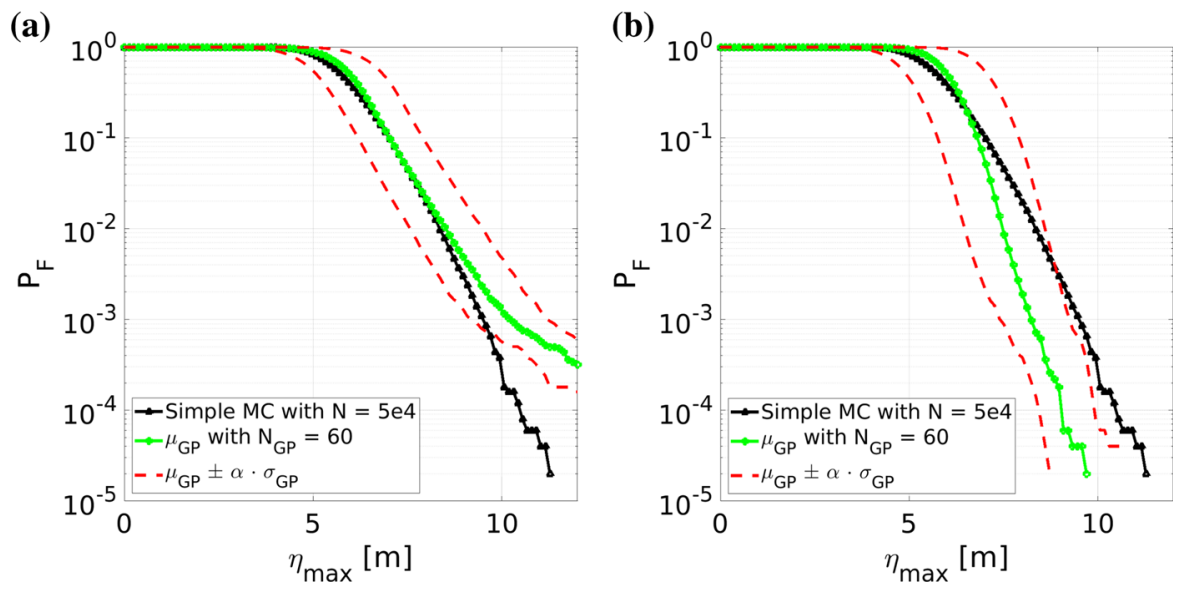

Fig. 3 The initial estimation of the short-term exceedance probability $\hat{P}_{\mathrm{F}}$ for the KdV22 model using the Gaussian surrogate model with $\mathbf{a}$ the quadratic trend, and $\mathbf{b}$ the linear trend

we can select $N_{\mathrm{GP}}=60$ wave input samples randomly. As seen in Fig. 1a, we choose to have the wave input samples equally spread.

For each wave input, we evaluate the KdV22 model and collect the output value $\eta_{\max }$ to construct the training set $\mathcal{S}_{K}:=\left\{\left(K_{1-10, j}, \eta_{\max , \mathrm{j}}\right), j=1, \ldots, N_{\mathrm{GP}}\right\}$. Using the training set $\mathcal{S}_{K}$, we define the Gaussian process based on the anisotropic squared exponential kernel with the pure quadratic and the linear trend, respectively. We evaluate the $N=5 \cdot 10^{4}$ generated wave input samples to estimate the short-term exceedance probability as demonstrated in Fig. 3. Note that this part is low cost as we just need to evaluate Eq. (2.3) for the trained Gaussian process. The main cost for this part comes from the inverse of $\mathbf{K}$.

In Fig. 3, we plot the results obtained by the initial Gaussian process with the pure quadratic and linear trend with $N_{\mathrm{GP}}=60$. The performances of these processes are compared with the simple Monte Carlo method with $N=5 \times 10^{4}$ evaluations. As the KdV22 model is simple, we can generate $N=5 \times 10^{4}$ realizations within one day. The initial Gaussian models are inadequate for extreme events, with a pronounced discrepancy occurring for $P_{\mathrm{F}} \leq 10^{-2}$. Typical offshore reliability requirements for exceedance probabilities are in the range $10^{-4}$ to $10^{-2}$ [20]. The initial value of the stopping criterion in Eq. (2.8) is estimated at $\varepsilon_{9.45}^{\text {pure }}=10.96$ and $\varepsilon_{9.45}^{\operatorname{lin}}=10.96$, respectively, for each of the trends and for $\gamma=9.45 \mathrm{~m}$, see Fig. 3 . 

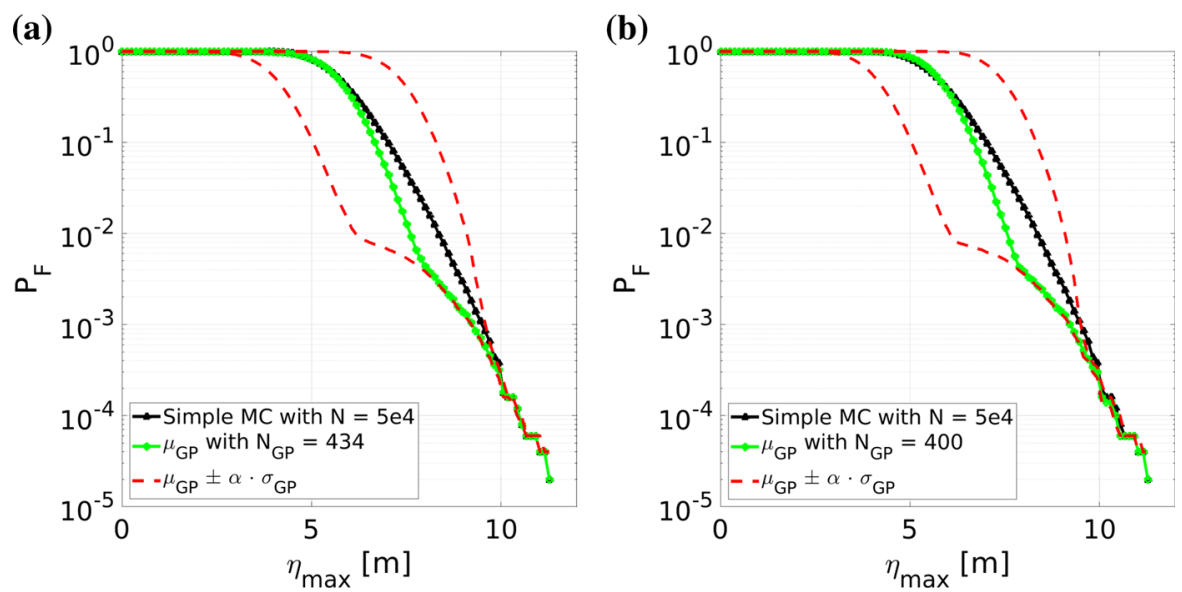

Fig. 4 The final estimation of the short-term exceedance probability $\hat{P}_{\mathrm{F}}$ for the KdV22 model using the Gaussian surrogate model with a the quadratic trend, and $\mathbf{b}$ the linear trend

Fig. 5 The mean-squared error (MSE) between the reference tail of the distribution generated with $N=5 \times 10^{4}$ and the corresponding approximations of Gaussian process regression as a function of the number of samples $N_{\mathrm{GP}}$. (Color figure online)

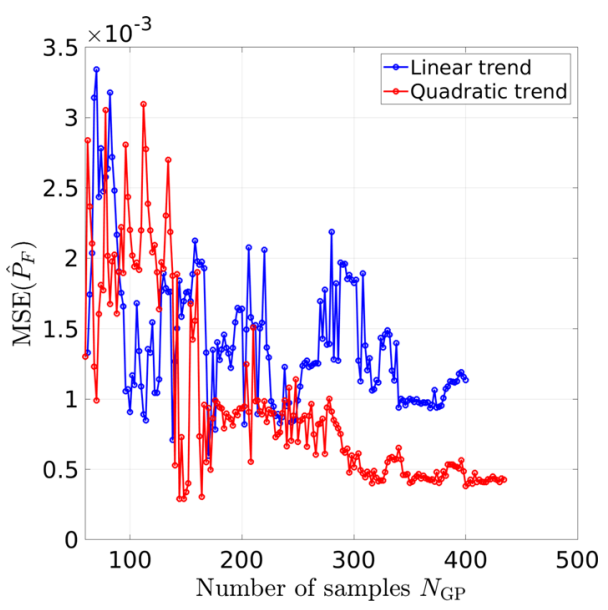

Figure 4 shows the final results after the learning process based on the $U$-design improvement (i.e., Algorithm 1) has terminated after reaching the threshold $\xi=2$. For the pure quadratic trend, we have $\varepsilon_{9.45}^{\text {pure }}=1.66$ at $10^{-3}$, while $\varepsilon_{9.45}^{\operatorname{lin}}=1$. The choice of the error threshold value of 2 seems reasonable in light of the typical levels of the exceedance probabilities $\left(10^{-4}\right.$ or $\left.10^{-3}\right)$. The pure quadratic trend requires more evaluations than the linear trend as shown in Fig. 5. However, the performance of the pure quadratic trend is better, with the mean squared error (MSE) less than $4 \times 10^{-4}$, while for the linear trend the MSE is less than $1.2 \times 10^{-3}$. By visually inspecting Fig. 4 and Table 1 , it is observed that the model with the pure quadratic trend performs better. The total number of evaluations of the KdV22 model for the pure quadratic trend is $N_{\mathrm{GP}}^{\text {pure }}=434$ and for the linear trend $N_{\mathrm{GP}}^{\text {lin }}=400$, which is around $0.87 \%$ of the total number of simple Monte Carlo evaluations. Mostly, the design samples are closer to the events with higher peaks, see Fig. 1. The simple Monte Carlo estimations are accurately recreated for extreme events, while significant differences for $P_{\mathrm{F}}>10^{-3}$ are seen. This illustrates the ability of the method to focus the approximation quality to a user-defined probability interval.

\subsection{Application to fully nonlinear wave propagation over a slope}

We next apply the classification approach with active learning to compute the short-term exceedance probability for wave propagation over a slope with a fully nonlinear model, OceanWave3D [27]. OceanWave3D is a finite 
Table 1 Estimation of the maximum wave crest $\eta_{\max }[\mathrm{m}]$ using Gaussian process regression with the $U$-function for the KdV22 model and different exceedance levels

\begin{tabular}{lllll}
\hline Method & $\hat{P}_{\mathrm{F}}=10^{-3}$ & $\hat{P}_{\mathrm{F}}^{+}=10^{-3}$ & $\hat{P}_{\mathrm{F}}=10^{-4}$ & $\hat{P}_{\mathrm{F}}^{+}=10^{-4}$ \\
\hline Simple MC & 9.48 & - & 10.48 & - \\
Pure quadratic & 9.38 & 9.67 & 10.48 & 10.54 \\
Linear & 9.22 & 9.55 & 10.42 & 10.54 \\
\hline
\end{tabular}

(a)

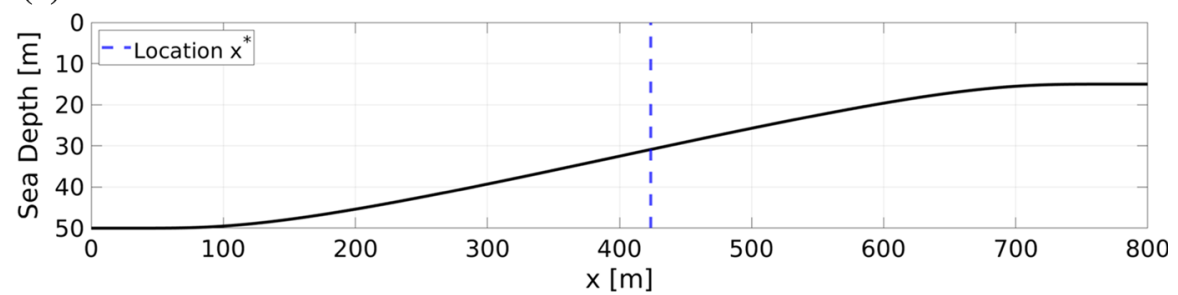

(b)

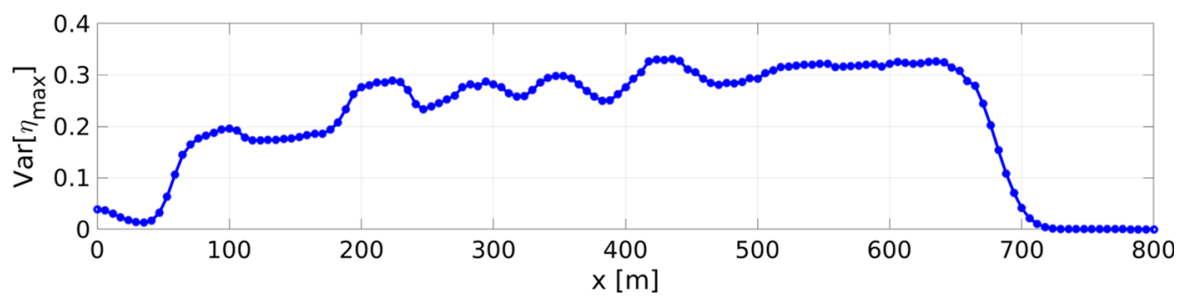

Fig. 6 a Illustration of the sloping seabed with the reference location as the blue line. b The expectation of the squared deviation of $\eta_{\max }$ from its mean, i.e., the variance for each spatial position

difference potential flow wave model based on the Laplace equation with kinematic and dynamic free surface boundary conditions. An ad hoc wave breaking filter is included within the model. A complete derivation of the equations can be found in [35]. It is recognized as a high-order model as it utilizes the high-order discretization, which enables to propagate waves over a significant distance with unsubstantial numerical errors [36].

We establish our numerical experiment on the benchmark example of OceanWave3D defined for random waves over a slope [32]. MATLAB codes for this numerical experiment can be found at https://github.com $/ \mathrm{ksehic/}$ OCW3D-F90-UQProbe in the folder UQ-Packages. The sea state parameters for the JONSWAP spectrum are $H_{\mathrm{S}}=4 \mathrm{~m}$ and $T_{\mathrm{P}}=9 \mathrm{~s}$. The computation is additionally simplified as it includes 137 cells with a time duration of $256 \mathrm{~s}$ and $800 \mathrm{~m}$ of the spatial domain. The initial sea depth is $50 \mathrm{~m}$ and gradually decreases to a shallow region of $15 \mathrm{~m}$ depth. The reference location is at the depth of approximately $30 \mathrm{~m}$, see Fig. 6a. Figure $6 \mathrm{~b}$ shows the variance estimations for different spatial locations using the initial runs of OceanWave3D. We choose the reference location for the short-term exceedance probability $\hat{P}_{\mathrm{F}}$ with the highest estimated variance. It is interesting to notice that the most uncertain location for this example and data is at the depth of $h \approx 30 \mathrm{~m}$, which is a depth reduction of $40 \%$.

The procedure is the same as previously described for the KdV22 shallow-water model. First, we generate and classify $N=5 \times 10^{4}$ wave input samples with the predefined sea state $\left(H_{\mathrm{S}}=4 \mathrm{~m}\right.$ and $\left.T_{\mathrm{P}}=9 \mathrm{~s}\right)$ using $K_{1-10}$ as demonstrated in Fig. 7. To define the training set, we randomly select initial $N_{\mathrm{GP}}=60$ wave input samples for which we evaluate OceanWave3D as we see in Fig. 7a. Using this training set, we define the Gaussian process with the anisotropic squared exponential kernel function based on the pure quadratic regression. Next, we estimate the short-term exceedance probability as the sample mean with the indicator function using $N$ generated samples as shown in Fig. 8a. Initially, we need to select a failure threshold for a numerical model and active learning. Thus, we select the failure threshold $\gamma=4 \mathrm{~m}$ as $H_{\mathrm{S}}$. The initial error measure equation (2.8) attains the value $\varepsilon_{4}=5.4$, see 

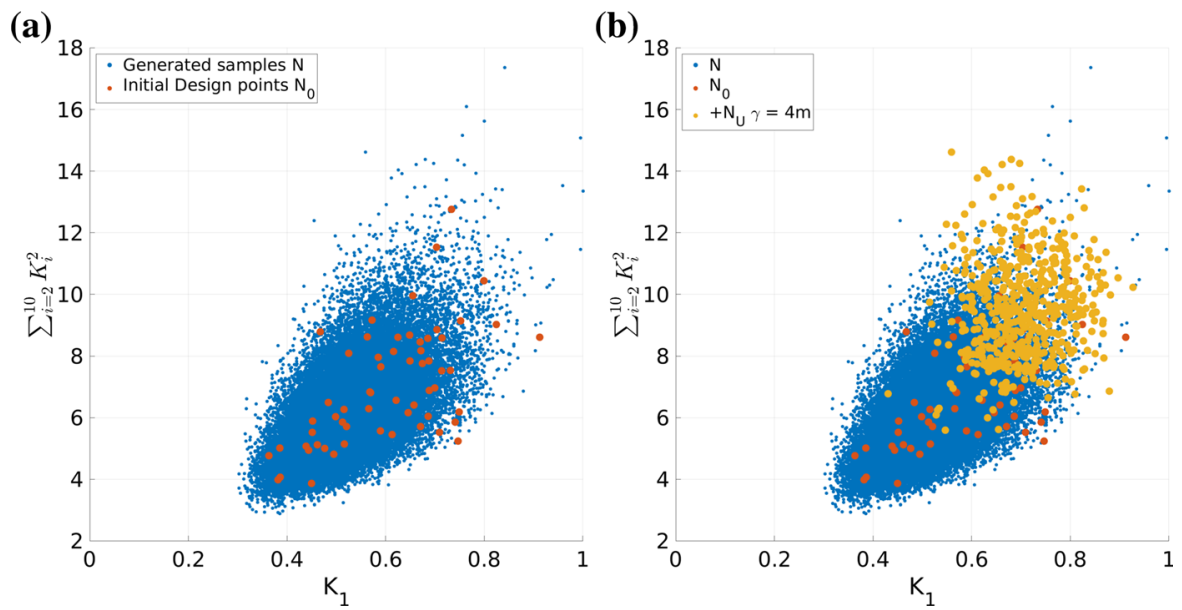

Fig. 7 The wave generation classification data generated based on $H_{\mathrm{S}}=4 \mathrm{~m}$ and $T_{\mathrm{P}}=9 \mathrm{~s}$ with a initial training set $N_{0}$, and $\mathbf{b}$ additional $U$-samples $N_{\mathrm{U}}$ for $\gamma=4 \mathrm{~m}$. (Color figure online)
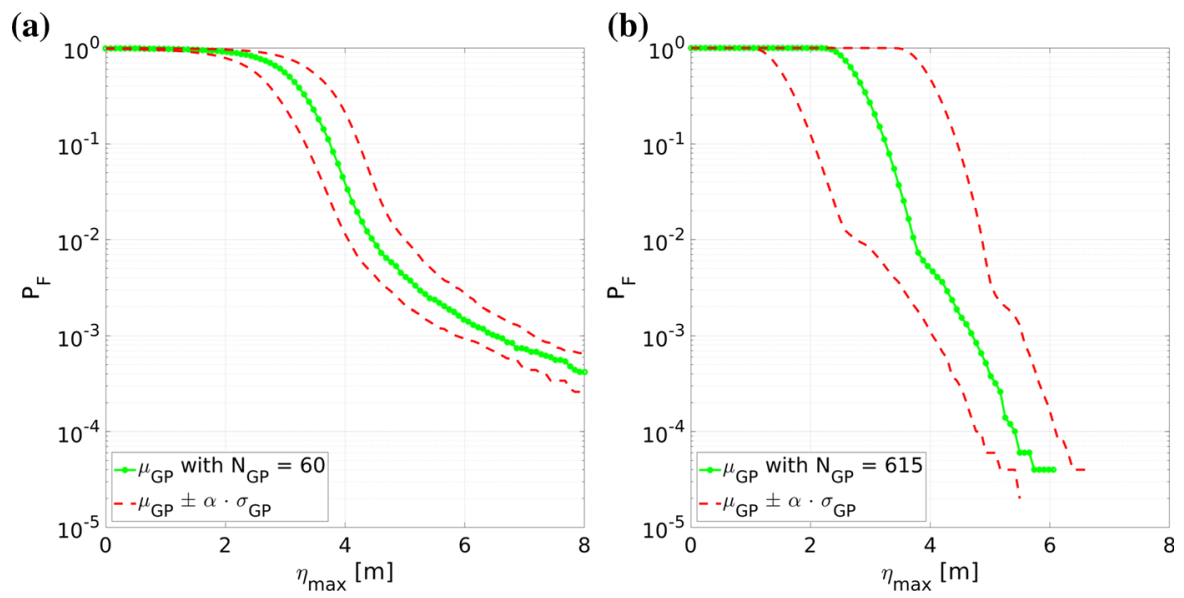

Fig. 8 The estimation of the short-term exceedance probability $\hat{P}_{\mathrm{F}}$ using the trained Gaussian process of OceanWave3D based on a $N_{\mathrm{GP}}=60$ initial design points, and $\mathbf{b} N_{\mathrm{GP}}=615$ with the additional $U$-design points for $\gamma=4 \mathrm{~m}$

Table 2. This error measure is in this case too optimistic, in that the confidence interval is significantly narrowed with the error measure close to the predefined stopping criterion of $\xi=2$. It is a clear example that one should consider the confidence interval of a Gaussian process with skepticism. We can see in Fig. 8a that the tail of the distribution $P_{\mathrm{F}} \geq 10^{-2}$ is extremely heavy. The prediction is similar to the initial Gaussian prediction of the KdV22 model, see Fig. 3, which diverges significantly from its reference solution. If the prediction is true, additional carefully selected new wave input samples can only improve the stability of the Gaussian process. However, when the Gaussian process starts to learn and explore the probability space actively, the error measure $\varepsilon_{4}$ starts to converge to a value that is higher than the initial value. During the learning process (i.e., Algorithm 1), in each iteration we add five new wave input samples with lowest $U$-values. Numerical evaluations can be run in parallel once optimal wave input samples are selected. After 615 evaluations, we stop the algorithm and find the lowest error measure $\varepsilon_{4}=95.7$ since the beginning of the learning process, see Fig. $9 \mathrm{~b}$. As expected, the wave input samples have mostly populated the area far from the origin as illustrated in Fig. 7.

Visual inspection of Fig. 9a reveals that the Gaussian process reproduces the tail of the distribution. The Gaussian prediction of the short-term exceedance probability resembles typical offshore exceedance probabilities and is similar to what we have found with the KdV22 model. Still, the error measures are significant with high uncertainties 
Table 2 The convergence rate based on the error measure $\varepsilon_{\gamma}$, Eq. (2.8), at different failure levels $\gamma$ and training sets for OceanWave3D

\begin{tabular}{lllrl}
\hline Training set & $\gamma=3.45 \mathrm{~m}$ & $\gamma=3.75 \mathrm{~m}$ & $\gamma=4 \mathrm{~m}$ & $\gamma=4.6 \mathrm{~m}$ \\
\hline$N_{\mathrm{GP}}=60$ & 2 & 3.2 & 5.4 & 2.9 \\
$N_{\mathrm{GP}}=615$ & 23.9 & 90 & 95.7 & 32.4 \\
$N_{\mathrm{GP}}=845$ & 30 & 1.1 & 0.1 & 0 \\
\hline
\end{tabular}

(a)

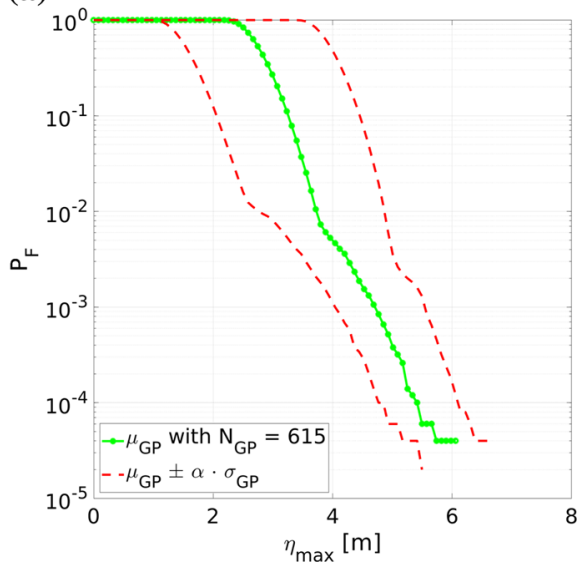

(b)

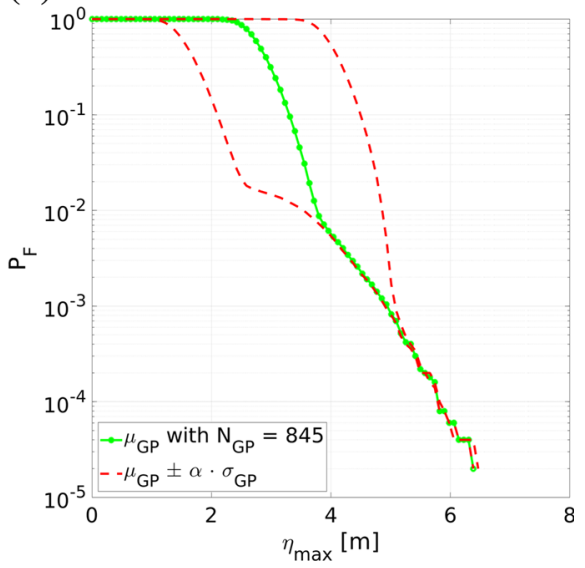

Fig. 9 The estimation of the short-term exceedance probability $\hat{P}_{\mathrm{F}}$ using the trained Gaussian process of OceanWave3D based on a $N_{\mathrm{GP}}=615$ training wave input samples for $\gamma=4 \mathrm{~m}$, and $\mathbf{b} N_{\mathrm{GP}}=845$ with the additional $U$-design points after we change the failure threshold to $\gamma=4.6 \mathrm{~m}$

Table 3 Estimation of the maximum wave crest $\eta_{\max }[\mathrm{m}]$ using Gaussian process regression with the $U$-function for a fully nonlinear wave model, OceanWave3D

\begin{tabular}{lllcc}
\hline Training set & $\hat{P}_{\mathrm{F}}=10^{-3}$ & $\hat{P}_{\mathrm{F}}^{+}=10^{-3}$ & $\hat{P}_{\mathrm{F}}=10^{-4}$ & $\hat{P}_{\mathrm{F}}^{+}=10^{-4}$ \\
\hline$N_{\mathrm{GP}}=60$ & 6.54 & 7.15 & 10.06 & 10.9 \\
$N_{\mathrm{GP}}=615$ & 4.69 & 5.52 & 5.41 & 6.1 \\
$N_{\mathrm{GP}}=845$ & 4.93 & 5.05 & 5.8 & 5.81 \\
\hline
\end{tabular}

in predictions, see Table 3 for $N_{\mathrm{GP}}=645$. We can see that the failure threshold for $10^{-3}$, which is our goal, will likely be higher than $4 \mathrm{~m}$. Thus, we change the predefined failure threshold to $4.6 \mathrm{~m}$ as this threshold may correspond to the exceedance order of $10^{-3}$ eventually.

We therefore iteratively include additional 230 evaluations based on the $U$-function and the new failure threshold for the learning process, see Fig. 10b. The Gaussian process continues to learn and improve the predictions until the error measure drops below the stopping criterion of $\xi=2$, see Table 3 for $N_{\mathrm{GP}}=845$. The trained Gaussian process eventually estimates the short-term exceedance probability with a significantly narrowed confidence interval for the tail, see Fig. 9. The tail of the distribution is, to a certain extent, what we would expect for this case. With the new failure threshold $\gamma=4.6$, the Gaussian process explores further in the tail as shown in Fig. 10b.

In Table 3, we present the results for the maximum crest elevation $\eta_{\max }$ at the depth of approximately $30 \mathrm{~m}$, for the exceedance probability levels $10^{-3}$ and $10^{-4}$. The maximum crest elevation for the exceedance level of $10^{-3}$ is $4.93 \mathrm{~m}$. The upper bound for the same level is recorded as $5.05 \mathrm{~m}$.

Using $N_{\mathrm{GP}}=845$ training evaluations, we can additionally examine the effect of the slope on the quantity of interest and classification parameters. In Fig. 11, we plot the normalized histograms at four different depths. It is 
(a)

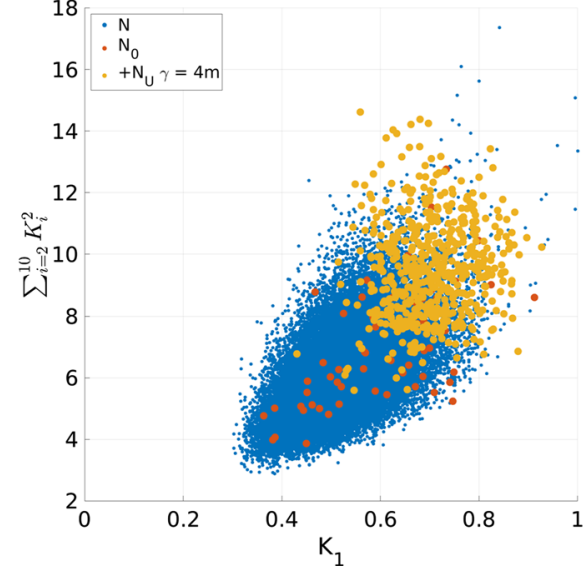

(b)

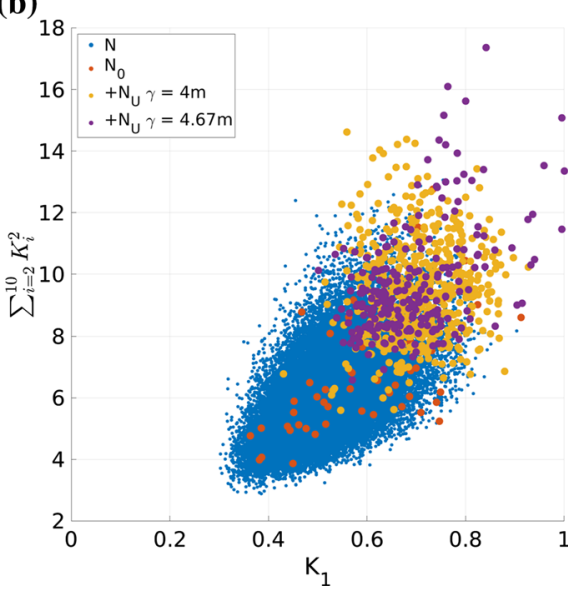

Fig. 10 The samples $N_{\mathrm{U}}$ selected by the $U$-function with the failure threshold $\mathbf{a} \gamma=4 \mathrm{~m}$, and $\mathbf{b} \gamma=4.67 \mathrm{~m}$. (Color figure online)

Fig. 11 The histograms for different depths using $N_{\mathrm{GP}}=845$ evaluations of OceanWave3D (a)
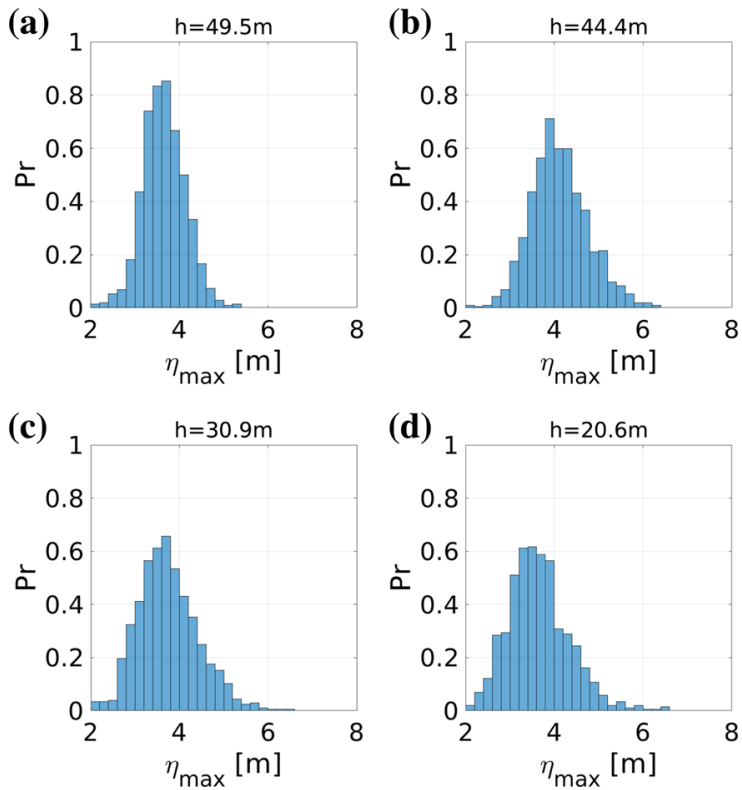

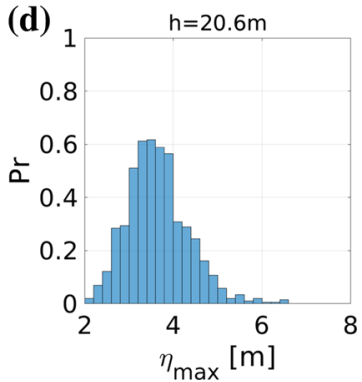

worth noticing how the slope, i.e., decreasing depth, causes the initially normal distribution at the depth of $50 \mathrm{~m}$ to change smoothly to a heavy-tailed distribution.

Figure 12 describes the global sensitivity for the classification parameters $K_{1-10}$ concerning the quantity of interest $\eta_{\max }$ at four different depths using the Pearson correlation measure. While the seabed is flat, the classification parameter $K_{1}$ related to the maximum wave height at $x=0$, the kurtosis $K_{4}$, and approximated entropy $K_{6}$ are the most significant parameters. As the depth decreases, the parameters related to the variance $K_{2}$, such as the root mean square, the time-series percentiles for $75 \%$ and $90 \%$, and the variance $K_{2}$ itself influence the maximum crest elevation more than the rest of the parameters. It is interesting to notice how the skewness and kurtosis lose their influence, while the wave input percentiles for extreme events gain significance with decreasing depth. The approximate entropy $K_{6}$, which has a significant negative correlation with the variance, becomes negligible as the sea depth decreases. This parameter generally quantifies the regularity and unpredictability of time-series variation. 
Fig. 12 Correlation analysis for different depths using $N_{\mathrm{GP}}=845$ evaluations of OceanWave3D
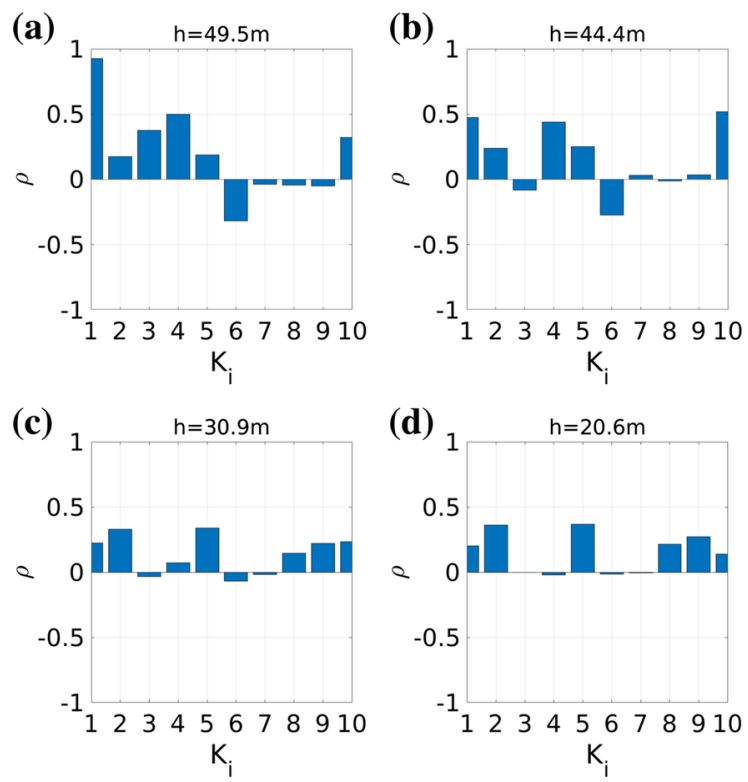

\section{Conclusion and further work}

When the high-dimensional input vector of Fourier coefficients is used to generate the wave input (i.e., timeseries), standard dimensionality reduction approaches do not provide a sufficient low-dimensional subspace to allow an efficient quantification of extreme events for nonlinear wave-propagation models with Gaussian process regression. Therefore, for a sequential design (i.e., active learning), we propose to employ feature extraction [26] to determine a sufficient low-dimensional representation based on time-series classification parameters. Initially, we generated different independent realizations of surface elevation required for the simple Monte Carlo estimation of the short-term exceedance probability. By using the generated data, we defined the classification parameters and selected a few evaluations to train a Gaussian process. The initial Gaussian process estimated the short-term exceedance probability for the maximum crest elevation at the reference point, which deviated significantly from the reference solution. Thereafter, the surrogate model was improved actively using the $U$-function until the confidence interval of predictions dropped below some prescribed stopping criterion for the predefined failure threshold. Using the $U$-function, the learning criterion emphasizes the predefined failure threshold and uncertain predictions. We demonstrated the applicability through two offshore problems, one of them involving a weakly nonlinear Kortewegde Vries model to reproduce simple shallow-water wave conditions and another the wave propagation over a slope for a fully nonlinear model, OceanWave3D. Our proposal employs less than $1.7 \%$ of the required Monte Carlo evaluations. As we based our approach on the generated data, the learning process is discrete and not optimal. The total number of evaluations can indeed be additionally reduced by continuous optimization.

We plan to examine how to optimally prepare the data for learning and establish classification parameters for extreme events. Using the Pearson correlation measure, we can build and explore which classification parameters we should choose to quantify extreme events accurately and efficiently. In the present study, we use the stationary description of the wave input (i.e., a time-series). Therefore, we shall next focus on moving statistics and their influence on extreme events. To provide a more systematic approach to determine the low-dimensional representation, we are determined to exploit multifidelity methods [37] in which we can use a low-cost numerical model based on a coarser mesh grid to define adequate classification parameters. We will focus also on real-time experiments to analyze the characteristics of the wave input that potentially generate large waves.

Acknowledgements This research was funded by the DeRisk project of Innovation Fund Denmark, Grant Number 4106-00038B. The authors very much appreciate the support by the Innovation Fund Denmark. 


\section{References}

1. Bigoni D, Engsig-Karup AP, Eskilsson C (2016) Efficient uncertainty quantification of a fully nonlinear and dispersive water wave model with random inputs. J Eng Math 101(1):87-113

2. Bredmose H, Dixen M, Ghadirian A, Larsen TJ, Schløer S, Andersen SJ, Wang S, Bingham HB, Lindberg O, Christensen E, Vested MH, Carstensen S, Engsig-Karup AP, Petersen OS, Hansen HF, Mariegaard JS, Taylor PH, Adcock TAA, Obhrai C, Gudmestad OT, Tarp-Johansen NJ, Meyer CP, Krokstad JR, Suja-Thauvin L, Hanson TD (2016) DeRisk-accurate prediction of ULS wave loads. Outlook and first results. Energy Procedia 90:379-387

3. Ghadirian A, Bredmose H (2019) Pressure impulse theory for a slamming wave on a vertical circular cylinder. J Fluid Mech 867:R1

4. Yildirim B, Karniadakis GE (2015) Stochastic simulations of ocean waves: an uncertainty quantification study. Ocean Model $86: 15-35$

5. Rackwitz R (2001) Reliability analysis—a review and some perspectives. Struct Saf 23:365-395

6. Owen AB (2013) Monte Carlo theory, methods and examples. Open Access

7. Li J, Xiu D (2010) Evaluation of failure probability via surrogate models. J Comput Phys 229(23):8966-8980

8. Schöbi R, Sudret B, Marelli S (2016) Rare event estimation using polynomial-chaos kriging. ASCE ASME J Risk Uncertain Eng Syst A Civ Eng 3(2):D4016002

9. Constantine PG (2015) Active subspaces: emerging ideas for dimension reduction in parameter studies. SIAM, Philadelphia

10. Nicodemi M (2012) Extreme value statistics. Springer, New York

11. Dematteis G, Grafke T, Vanden-Eijnden E (2018) Rogue waves and large deviations in deep sea. Proc Natl Acad Sci USA 115(5):855-860

12. Varadhan SRS (1984) Large deviations and applications. SIAM, Philadelphia

13. Risken H (1989) The Fokker-Planck equation methods of solution and applications, 2nd edn. Springer, Berlin, Heidelberg, New York

14. Mohamad MA, Sapsis TP (2018) Sequential sampling strategy for extreme event statistics in nonlinear dynamical systems. Proc Natl Acad Sci USA 115(44):11138-11143

15. Anastopoulos PA, Spyrou KJ, Bassler CC, Belenky V (2016) Towards an improved critical wave groups method for the probabilistic assessment of large ship motions in irregular seas. Prob Eng Mech 44(2016):18-27

16. Boccotti P (1983) Some new results on statistical properties of wind waves. Appl Ocean Res 5(3):134-140

17. Cousins W, Sapsis TP (2016) Reduced-order precursors of rare events in unidirectional nonlinear water waves. J Fluid Mech 790:368-388

18. Lindgren G (1970) Some properties of a normal process near a local maximum. Ann Math Stat 41(6):1870-1883

19. Tromans PS, Anaturk AR, Hagemeijer A (1991) A new model for the kinematics of large ocean waves-application as a design wave. In: Proceedings of the First International Offshore and Polar Engineering Conference, pp 64-71

20. Šehić K, Bredmose H, Sørensen JD, Karamehmedović M (2021) Activesubspace analysis of exceedance probability for shallowwater waves. J Eng Math 126: 1

21. Jolliffe I (2002) Principal component analysis, 2nd edn. Springer, New York

22. Sclavounos PD (2012) Karhunen-Loeve representation of stochastic ocean waves. Proc R Soc 468:2574-2594

23. Bouhlel M, Bartoli N, Otsmane A, Joseph Morlier J (2016) Improving kriging surrogates of high-dimensional design models by partial least squares dimension reduction. Struct Multidiscip Opt 53:935-952

24. Papaioannou I, Ehre M, Straub D (2019) PLS-based adaptation for efficient PCE representation in high dimensions. J Comput Phys 387:186-204

25. Gonzalez FJ, Balajewicz M (2018) Deep convolutional recurrent autoencoders for learning low-dimensional feature dynamics of fluid systems. CoRR abs/180801346

26. Esmael B, Arnaout A, Fruhwirth RK, Thonhauser G (2013) A statistical feature-based approach for operations recognition in drilling time series. IJCISIM 5(2150-7988):454-461

27. Engsig-Karup AP, Bingham H, Lindberg O (2009) An efficient flexible-order model for 3D nonlinear water waves. J Comput Phys 228:2100-2118

28. Rasmussen CE, Williams CKI (2006) Gaussian processes for machine learning. MIT Press, Cambridge

29. Shahriari B, Swersky K, Wang Z, Adams R, de Freitas N (2016) Taking the human out of the loop: a review of bayesian optimization. Proc IEEE 104(1):148-175

30. Gramacy RB, Apley DW (2015) Local gaussian process approximation for large computer experiments. J Comput Graph Stat 24:561-578

31. Naess A, Moan T (2012) Stochastic dynamics of marine structures. Cambridge University Press, Cambridge

32. Paulsen BT (2019) OceanWave3D-Fortran90. GitHub repos. https://github.com/boTerpPaulsen/OceanWave3D-Fortran90

33. Bredmose H (1999) Evolution equations for wave-wave interaction. Master's thesis, Technical University of Denmark, Lyngby, Denmark

34. Conrad PR, Marzouk YM, Pillai NS, Smith A (2016) Accelerating asymptotically exact MCMC for computationally intensive models via local approximations. J Am Stat Assoc 111(516):1591-1607

35. Engsig-Karup AP, Glimberg LS, Nielsen AS, Lindberg O (2013) Fast hydrodynamics on heterogenous many-core hardware. In: Couturier R (ed) Designing Scientific Applications on GPUs. Chap. 11. Taylor Francis, Abingdon, pp 251-294 
36. Schløer S, Bredmose H, Ghadirian A (2017) Experimental and numerical statistics of storm wave forces on a monopile in uni- and multidirectional seas. In: Proceedings of the ASME 2017 36th Int Conf Omae 10:OMAE2017-61676

37. Ullmann E, Papaioannou I (2015) Multilevel estimation of rare events. SIAM/ASA J UQ 3:922-953

Publisher's Note Springer Nature remains neutral with regard to jurisdictional claims in published maps and institutional affiliations. 